\title{
AD SHARING IN SOCIAL NETWORKS : ROLE OF USER DEFINED POLICIES
}

\author{
Venkata N Inukollu ${ }^{1}$ Sailaja Arsi ${ }^{1}$ Divya D Keshamoni ${ }^{2}$ \\ and Manikanta Inukollu ${ }^{3}$ \\ ${ }^{1}$ Department of Computer Science Engineering, Texas Tech University, USA \\ \{narasimha.inukollu, sailaja.arsi\}@ttu.edu \\ ${ }^{2}$ Rawls College of Business, Texas Tech University, USA \\ divya.keshamoni@ttu.edu \\ ${ }^{3}$ Department of Computer Science, Bhaskar Engineering College, India \\ mani.inukollueyahoo.com
}

\begin{abstract}
Security policies describe the demeanor of a system through specific rules and are becoming an increasingly popular approach for static and dynamic environment applications. Online social networks have become a de facto portal for Internet access for millions of users. Users share different content on social media sometimes which includes personal information.However, users entrust the social network providers with such personal information.Although social networking sites offer privacy controls, the sites provide insufficient controls to restrict data sharing and let users restrict how their data is handled and viewed by other users.To match the privacy demands of an online social network user, we have suggested a new security policy and have tested the policy successfully on various levels.
\end{abstract}

\section{KEYWORDS}

User defined policy, Social networking, Policy, User privacy.

\section{INTRODUCTION}

Security policy can be stated as what it means to be secure for a system, or an entity or an organization.The security policy should protect both people and information. Moreover, a security policy helps by minimizingthe risks and by compliance tracking with regulations and legislations.

The policies are used by different employees at different levels of the company. The classification can be done by different levels, such as management level, technical staff level and the end user level. Therefore, people play a vital role in explaining, maintaining, monitoring and using the policies. Thus, giving the user/consumer a high responsibility in defining a policy according to the user requirements would be appropriate for a successful product.

As per the survey results [10], $90 \%$ of the people are concerned about issues such as security, privacy, create fake accounts with once identification, children care, and people following them in their social networks. Of all the issues security and privacy is the major concern for nearly

David C. Wyld et al. (Eds) : COSIT, DMIN, SIGL, CYBI, NMCT, AIAPP - 2014

pp. 91-98, 2014. (C) CS \& IT-CSCP 2014

DOI : $10.5121 / \mathrm{csit} .2014 .4908$ 
$\sim 70 \%$ people. Online social networking sites are the most widely used means of communication. Thus, social media has become an integral part of modern life and so has sharing online content. $59 \%$ of people reported that they frequently share online content with others (Allsop, Bassett, and Hoskins 2007), the New York Times story receives a tweet once every four seconds. There are several advantages and disadvantages of using social networking. The main disadvantages of using any online social networking sites are:

a) users lose some privacy compared to not being on a social networking site.

b) users may take a step back while sharing some content such as advertisements.

c) Some users want to sustain a line between private and public life, but through social networking, the user may regret later for posting pictures that the user had thought were funny at that minute.

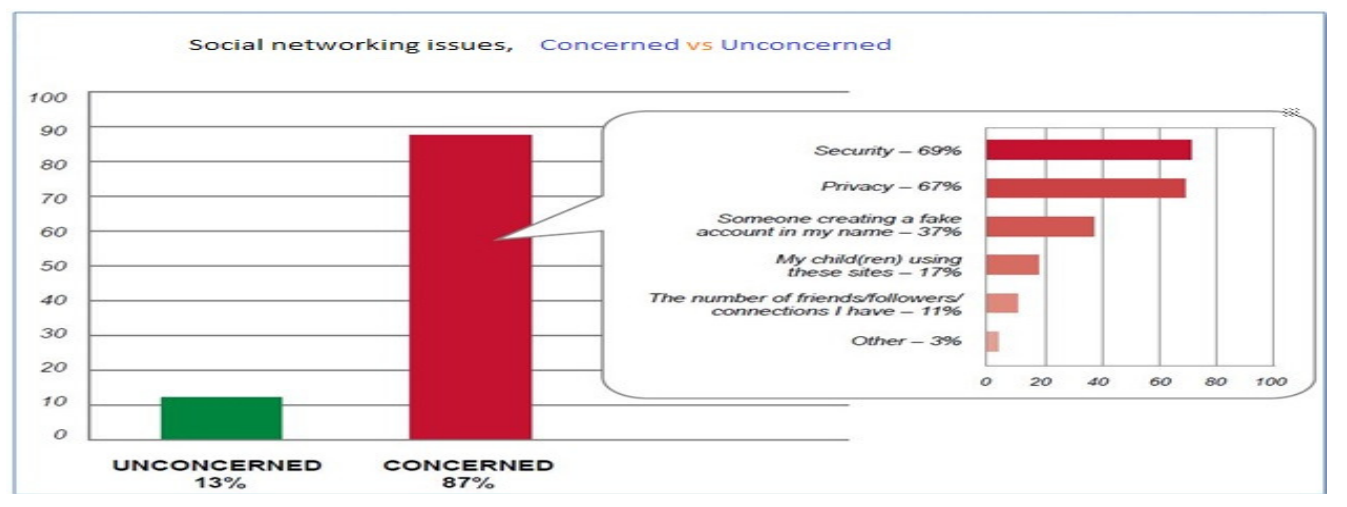

Figure (1): Concernd vs Unconcernedstatistics

So, there should be a common dynamic policy and that should be handled by the user. As the user is given the prime responsibility, user can maintain security and privacy accordingly.

Advantages of user defined security policies:

- user defined security policies address a wide variety of aspects of system security, such as access control, authentication, availability and auditing.

- user defined security policies provide a clear perspective of how to use data abstraction.

- user defined security policies can be applied to distributed and dynamic environment systems.

- user defined security policies providecustomer satisfaction by maintaining privacy and security.

- user defined security policy is a well defined communication paradigm.

- In user defined security policies, creation of object, subject, constraints and actions are all dynamic in nature and follow user satisfaction.

- user defined security policies, can resolve policy conflicts, as user defined security policies maintain separate entities belonging to their domains. User defined security policy has a clear perspective of how entity attributes are defined and exploited.

\section{MOTIVATION AND RELATED WORK}

An immense amount of work has been done along the user defined privacy by Randy Baden et al. [4], the work is focused on online social networking. In this paper, the authors have introduced a new model called as persona which acts as an online social network that puts policy decisions in 
the hands of the users. Persona includes attribute-based-encryption, traditional public key cryptography, and an automated key management mechanism to translate between the two cryptosystems. The persona is a well-defined model to achieve privacy and also provides userdefined access controls needed in online social networking.

Paul Ashley et al. [5], defines a fine grained privacy model called as Platform for the Enterprise Privacy Practices (E-P3P). The E-P3P privacy policy defines, on certain data categories the actions to be performed by the data users. This paper has given a well defined constructed model and semantics for E-P3P. The model comprises of formal semantics of simple requests, compound requests, language details, deployment mapping, defining conditions, authorization algorithm, and related interfaces. This paper has strongly stated that formalized and strictly enforced privacy practices enable enterprises to provide the level of privacy promised using privacy statements.

Oracle $10 \mathrm{~g}$ Enterprise Manager had released a step by step example for creating user defined policies and metrics [6]. Oracle Enterprise Manager in its own way created and modified tables to provide a simplified user defined policy and user defined metrics that has a user defined policy group interface.

Dasan in [7] explained an automatic method and system for retrieving information based on a user-defined profile such as personalized newspaper, wherein the user defined profile identifies information which is of interest to the user. Dasan has provided well-defined models for userdefined profile systems.

Schreckling et al. [8] introduced a real-time monitoring and enforcement framework for Android called Kynoid. Kynoid is based on user-defined security policies that are defined for data-items. The article on Kynoidmainly focuses on android framework, but the article does not mention whether kynoidcan be applied to different fields, frameworks/ platforms.

\section{ROLE OF USER DEFINED SECURITY IN AD MARKETING}

Consumers signal their identities through brands and products [11,12], or even restore their original self-view through brands [13].Companies often create online ad campaigns or encourage consumer-generated content in the hope that people will share this content with others, but some of these efforts take off while others fail.Despite the fact that viral marketing and advertising can be a successful means of marketing communication, there is still a limited understanding of how it works and why consumers' share online content[14].

Previous research indicates that consumers tend to share advertising messages that they find entertaining, informative, titillating, or shocking-that is, messages that evoke strong emotional responses [14].This research proposes that among various factors, Privacy controls for sharing and receiving content for different types of products(Public vs Privately consumed products) are important drivers of social transmission of information on the social media.

Publicly consumed products are those that are seen by others when being used, privately consumed products are ones that are not seen during the consumption process with the possible exception of the user. Research by [15] suggests that if consumers can be assured of their privacy, firms can use personalization of ads to generate higher click-through rates. According to [16], knowing that our consumption decisions are going to be subject to public scrutiny will influence our consumption choices. Similarly, individuals might be concerned about the information / advertisements that they share online because of the desire to be evaluated favorably by others. Individuals might sometimes feel compelled to switch away from sharing 
advertisements/content (of privately consumed goods). For e.g. hair fall products, foot fungus cream) because of how they expect to be perceived by others. Hence it can be proposed that control over personalized sharing and receiving of particular ads (especially for products linked to private consumption) increases the likelihood to share ads. Hence secure sharing plays a vital role in business development.

Eg: Presently Facebook displays several advertisements on the right hand corner of the page and users do not have the authority to share those advertisements. Also videos shared on Facebook by an individual are available publicly and potentially to all the friends in the users list. The current research examines if personalized sharing and receiving advertisements in private will boost individuals to partake in more number of advertisements online especially on Facebook. For e.g. If an ad about a fairness product appears on my fb page and if I would relish to share this ad only with one of my friends who is withal probing for more preponderant fairness products and if both of us have control over sharing/receiving the information without other friends on our face book knowing about it,it would enhance privacy.This would increase the number of ads clicked and shared.

\section{USER DEFINED SECURITY POLICIES}

One of the key foundations of a secure computer system is the security policy [1]. A security policy is a set of objectives, rules of behavior for users and administrators, and requirements for system configuration and management that collectively are designed to ensure security. Security policies can be expressed by associating security labels with either the data or actions that protect the system, as defined by Swamy et al. [2]. Based on the available literature, the following observations were made:

a) For each system's environment, assigning an individual policy to the system would be inappropriate because, for a particular system, there can be different environments, such as login authorization module, privileges module, malware defense module, account monitoring and control module, and testing module and for each environment it is not possible to have an individual policy applied to it.

b) For the entire system, the specification languages use only the common policies such as authorization, prohibition, obligation, delegation, information filtering, and refrain policies. All the environments within the system are explained using those common policies. There is no scope for policy extendibility.

From the above observations, user defined security policies are an arena that has not been handled and which needs great attention. User defined security policy means defining and implementing the system's scenario, according to the user. The user is afforded a main/primary role and the user takes the responsibility to ensure the security and privacy of the system. Currently, in that respect is the rapid growth in computer and online network resource utilization.In these circumstances, privacy is of top priority to preserve.

If users have a policy implementation scope, a user can develop the system according to the requirements while ensuring privacy. If such a user defined security policy comes into existence, then there will be many advantages for businesses, industries and organizations.From the table, if observed, there were several instances which were not handled by the existing policies. For those instances/environments we can assign the user defined security policy and let users implement their own policy, which handles both security and privacy of the system. 


\section{SECURE AD SHARING FRAMEWORK}

Ad marketing plays a vital role in social networking application in the context of revenues. The content on the social network consists of various kinds of data, such as personal, professional, and location based, but the current frameworks don't provide much needed privacy and security for the personal data. The only private communication that exists in the present social networking system is through mail Communication. In order to provide more privacy and security, we introduce a new component in the existing framework as user defined view where a user can have more control on his/her personal data. And we also introduce the new component as secure share where user can share his content with specific people or group of people. Currently social network applications provide sharing of information which can be viewed by public.(other than mail communication).

Figure (2) shows the social network application framework with the new components. User network shows all the data associated with the user account, such as photos, videos, social network, and text messages. In this level we introduce a new component called secure share where a user can share his/her private/personal information with specific people or group of people. All the user activities are carried out by the pre-defined access control polices in the social network system. In the view level, we have introduced one more new component called user-defined views, where user can see their own personal data, Shares (such as posts, messages, mail communication, multimedia sharing, ad sharing) that are shared by other users in his/her social network.

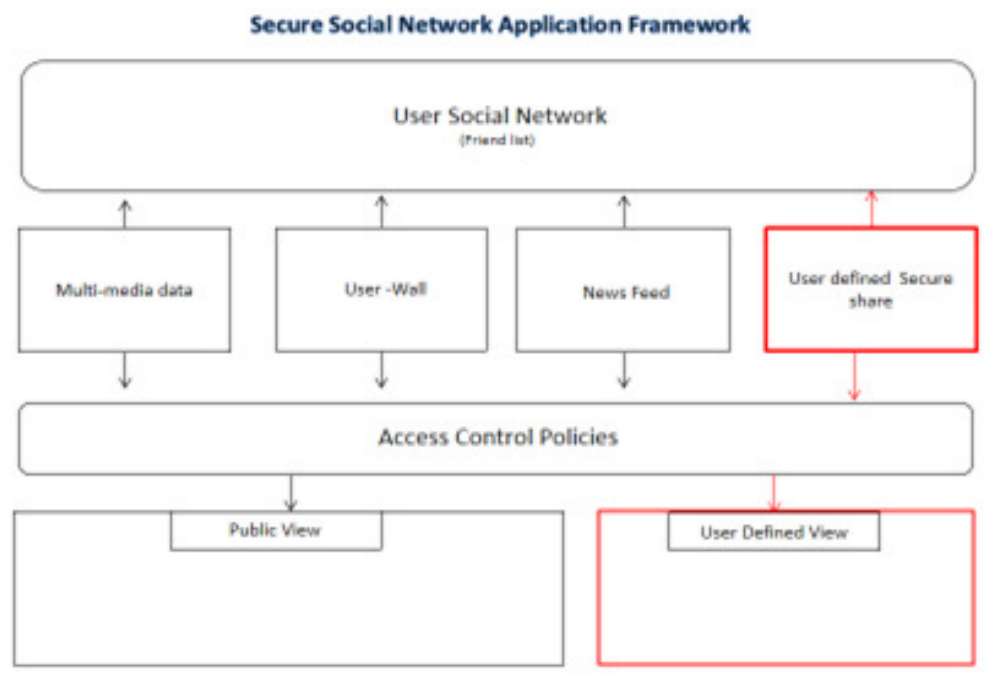

Figure (2): User defined components in Social network

\section{CASE STUdY: FACE BOOK}

Online social networks have become a de facto portal for Internet access for millions of users. Users mostly share private content such as personal information, photographs, gender preference, marital status, political and religious views, and identity of friends, occupations and phone numbers with their friends [3]. However, users entrust the social network providers with such personal information. Although social networking sites offer privacy controls, the sites provide insufficient controls to restrict data sharing and to let users restrict how their data is managed and viewed by other users. In this aspect, social networking sites lack the privacy of users. On high profile web sites the leakage of personal information [4] has heightened web user's privacy concerns. In that location requires to be an approach to prevent unintended leakage and 
manipulation of sensitive data, and that could offer strong guarantees, that deployed applications respect privacy and protection policies. To meet the privacy needs of an online social network, there should be a policy that puts the decision in the hands of the users which is addressed as 'user defined security policy'.

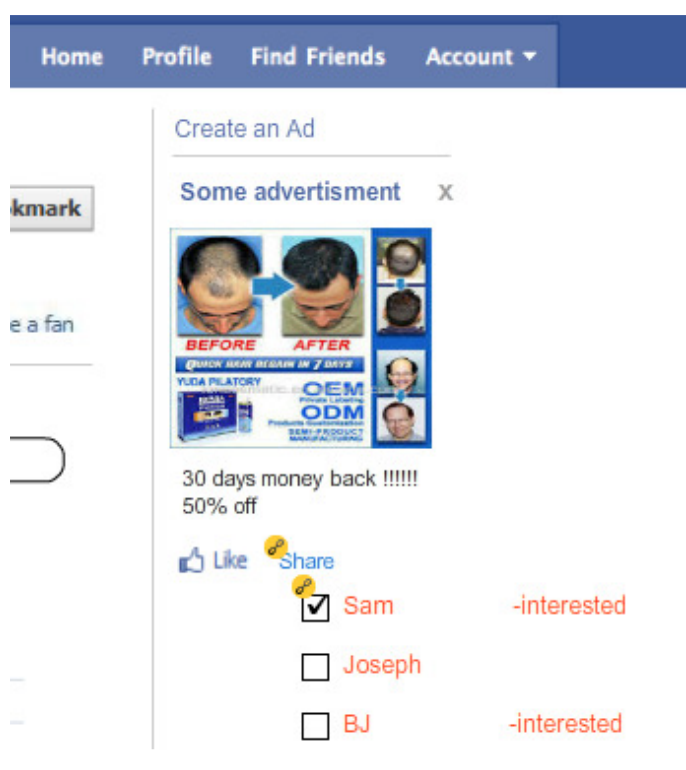

\section{Co to Application}

Add to my Page

Suggest to Friends

Block Application

Secure Ad feeds
1. Aging Cream by XX Shared by James

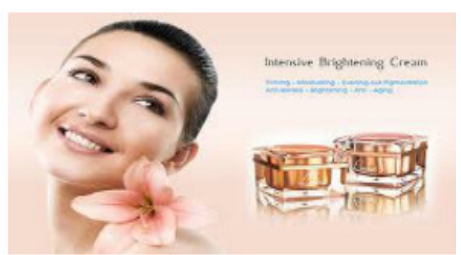

2. Hair loss products Shared by Sam

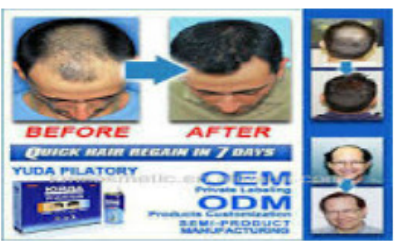

Figure (3): Facebook plug-in

As privacy is becoming a competitive advantage, enterprises need to secure user's personal information against both malicious privacy breaches and unintended data flows due to carelessness [3]. The main goal is to restrict the information shared with friends (in social network terms) to what might be appropriate.

In Facebook when an individual desires to share something, the options are limited, i.e. a user cannot share the data securely and thus this leads to exposure of user's personal interests/information such as the individual's interest in sharing a particular type of content in this instance of communion.In figure(3), we have implemented in face book, where a user has the 
freedomto share the data only with desired people and those contents can be viewed in secure ad feeds as shown in the figure(3), and this entire process is carried out in the user defined view.

\section{EXPERIMENTAL RESULTS}

We have implemented these components in the Facebook social network plugin and conducted a survey to validate our propositions.To our surprise every user in the survey liked the concept of user defined view and securelyshare, which shows that people are more concerned about the privacy and security of their personal and professional data. $70 \%$ people voted for ad sharing through secure share and $30 \%$ people votedfor personal data sharing through secure share. Additionally, from the survey we observed that the secure sharingof advertisements influences people in terms of buying products.

\section{SUMMARY}

Social networks have various issues in terms of privacy and security of the users personal and professional data. In order to provide additional security and privacy, we have introduced two new components, namelysecure share and user defined view in the existing social network framework and studied experimental results with the help of face book plug-in. As per the survey, user defined view provides much needed privacy to the users and secure share helps to improve their privacy and social communication. The research thus proposes that Social media users' message-sharing behaviors are motivated by the need for secure sharing. The social process of sharing an online advertising message shapes and helps express the consumers' sense of self, such that it influences which messages consumers are most likely to share with others. Thus, advertisements of privately consumed goods are passed on to friends and peers under shared privacy controls As per the survey results secure sharing exponentially increases the sharing of advertisements on social media and also helps the marketers to reach potential customers. This study contributes greatly to marketing practice as well. The ability to create and replicate successful viral advertising campaigns still remains challenging for online marketers. For every advertisement that successfully generates viral buzz, dozens fizzle. For the most part, Social media marketing practitioners still struggle to exploit an opportunity that has tantalized them for more than a decade. Any new insight into why consumers share some messages, but not others is thus significant and hence this research contributes significantly to both academic research and marketing practices.

\section{REFERENCES}

[1] Kuhnhauser W.E., "A Paradigm For User-Defined Security Policies," Proceedings of the Reliable Distributed Systems, 1995, Proceedings of the 14th Symposium, Bad Neuenahr, Sep 13-15, 1995, pp. 135-144.

[2] Swamy N., Corcoran B.J., and Hicks M., "FABLE: A Language for Enforcing User-defined Security Policies," Proceedings of the Security and Privacy, 2008, SP 2008, IEEE Symposium, Oakland, CA, May 18-22, 2008, pp. 369-383.

[3] Preibusch S., "Information Flow Control for Static Enforcement of User-Defined Privacy Policies," Proceedings of the Policies for Distributed Systems and Networks (POLICY), 2011 IEEE International Symposium, Pisa, Jun 6-8, 2011, pp. 133 - 136.

[4] Baden R., Bender A., Spring N., Bhattacharjee B., and Starin D., "Persona: An Online Social Network with User-Defined Privacy," Proceedings of the SIGCOMM'09, Barcelona, Spain, Aug 1721, 2009.

[5] Ashley P., Hada S., Karjoth G., and Schunter M., "E-P3P Privacy Policies and Privacy Authorization," Proceedings of the WPES'02, Washington, DC, USA, Nov 21, 2002.

[6] Oracle Enterprise manager 10g, "Step By Step Example for Creating User Defined Policies and Metrics". 
[7] Dasan V.S., "United States Patent," Jun 2, 1998.

[8] Schreckling, D., Posegga J., Köstler J., and Schaff M., "Kynoid: Real-Time Enforcement of FineGrained, User-Defined, and Data-Centric Security Policies for Android," Proceedings of the 6th IFIP WG 11.2 International Workshop, WISTP 2012, Egham, UK, Jun 20-22, 2012, pp. 208-223.

[9] Aaker, Jennifer L. (1999), "The Malleable Self: The Role of Self-Expression in Persuasion," Journal of Marketing Research, 36 (1), 45-57.

[10] Privacy stastics, http://www.welivesecurity.com/2011/06/22/linkedin-privacy/

[11] Kleine III, Robert E., Susan Schultz Kleine, and Jerome B. Kernan. "Mundane consumption and the self: a social-identity perspective." Journal of Consumer Psychology 2.3 (1993): 209-235.

[12] Laverie, Debra A., Robert E. Kleine III, and Susan Schultz Kleine. "Reexamination and extension of Kleine, Kleine, and Kernan's social identity model of mundane consumption: The mediating role of the appraisal process."Journal of Consumer Research 28.4 (2002): 659-669.

[13] Gao, Leilei, S. Christian Wheeler, and Baba Shiv. "The "shaken self": Product choices as a means of restoring self-view confidence." Journal of Consumer Research 36.1 (2009): 29-38.

[14] Dobele, Angela, et al. "Why pass on viral messages? Because they connect emotionally." Business Horizons 50.4 (2007): 291-304

[15] Goldfarb, Avi, and Catherine E. Tucker. "Privacy regulation and online advertising." Management Science 57.1 (2011): 57-71.

[16] Ratner, Rebecca K., and Barbara E. Kahn. "The Impact of Private versus Public Consumption on Variety-Seeking Behavior." Journal of Consumer Research29.2 (2002): 246-257. 